\title{
Generalized Fitch Graphs III: Symmetrized Fitch maps and Sets of Symmetric Binary Relations that are explained by Unrooted Edge-labeled Trees
}

\author{
Marc Hellmuth $^{1} \quad$ Carsten R. Seemann ${ }^{2,3}$ \\ Peter F. Stadler ${ }^{2-\left.6\right|^{*}}$ \\ 1 Department of Mathematics, Stockholm University, Sweden \\ 2 Max Planck Institute for Mathematics in the Sciences, Leipzig, Germany \\ 3 Bioinformatics Groups, Leipzig University, Germany \\ 4 Institute for Theoretical Chemistry, University of Vienna, Wien, Austria \\ 5 Facultad de Ciencias, Universidad Nacional de Colombia, Bogotá, Colombia \\ 6 The Santa Fe Institute, Santa Fe, United States
}

received $21^{\text {st } J a n . ~ 2020, ~ r e v i s e d ~} 21^{\text {st }}$ Jan. 2021, accepted $10^{\text {th }}$ May 2021.

\begin{abstract}
Binary relations derived from labeled rooted trees play an import role in mathematical biology as formal models of evolutionary relationships. The (symmetrized) Fitch relation formalizes xenology as the pairs of genes separated by at least one horizontal transfer event. As a natural generalization, we consider symmetrized Fitch maps, that is, symmetric maps $\varepsilon$ that assign a subset of colors to each pair of vertices in $X$ and that can be explained by a tree $T$ with edges that are labeled with subsets of colors in the sense that the color $m$ appears in $\varepsilon(x, y)$ if and only if $m$ appears in a label along the unique path between $x$ and $y$ in $T$. We first give an alternative characterization of the monochromatic case and then give a characterization of symmetrized Fitch maps in terms of compatibility of a certain set of quartets. We show that recognition of symmetrized Fitch maps is NP-complete. In the restricted case where $|\varepsilon(x, y)| \leq 1$ the problem becomes polynomial, since such maps coincide with class of monochromatic Fitch maps whose graph-representations form precisely the class of complete multi-partite graphs.
\end{abstract}

Keywords: Labeled Trees, Fitch Relations, Symmetrized Maps, Splits and Quartets, Recognition Algorithm, NPcompleteness, Phylogenetics

\section{Introduction}

Labeled phylogenetic trees are a natural structure to model evolutionary histories in biology. The leaf set $L$ of the tree $T$ correspond to currently living entities, while inner nodes model the branching of lineages that then evolve independently. Labels on vertices and edges annotate further details on evolutionary events.

* Supported in part by the Deutsche Forschungsgemeinschaft grant STA 850/49-1

ISSN 1365-8050 @ 2021 by the author(s) Distributed under a Creative Commons Attribution 4.0 International License 

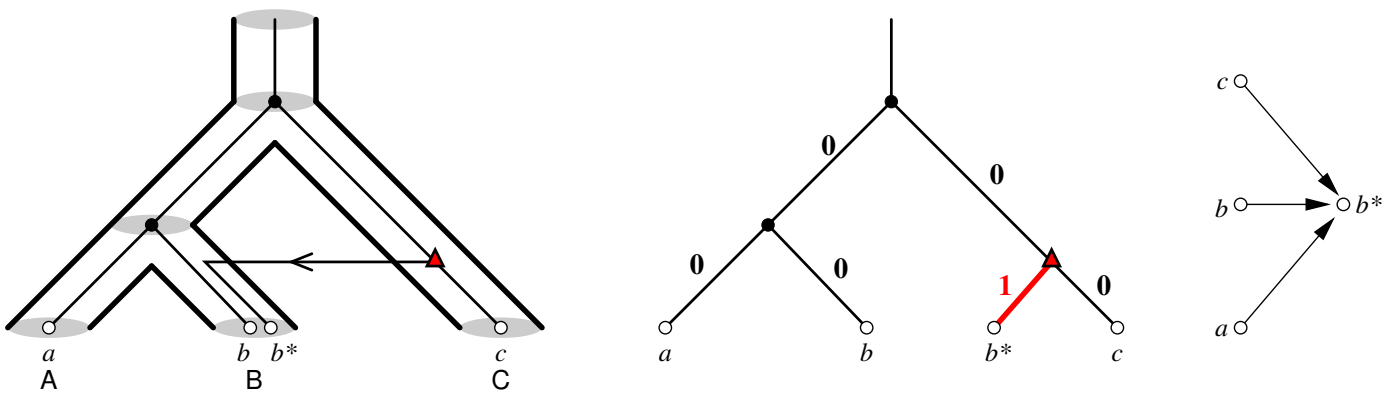

Fig. 1: The evolution of gene families is modeled as an embedding of a gene tree (thin lines, and r.h.s. panel, with four genes $a, b, b^{*}$ and $c$ ) into a species tree (shown as tubes with fat outlines with three species $\mathrm{A}, \mathrm{B}$, and $\mathrm{C}$ ). At each speciation (gray ellipses), each gene present in the genome is transmitted into both descending lineages, corresponding to a speciation even in the gene tree (shown as •). Horizontal gene transfer consists in a duplication of a gene, one copy of which "jumps" into a different lineage. The corresponding edge in the gene tree is marked with label 1. The graph representation of the directed Fitch relation $\rightarrow=\left\{\left(a, b^{*}\right),\left(b, b^{*}\right),\left(c, b^{*}\right)\right\}$ is shown on the right hand side. The underlying symmetrized Fitch relation $\sim$ is obtained from $\rightarrow$ by adding $(x, y)$ to $\sim$ if and only if $(x, y) \in \rightarrow$ or $(y, x) \in \rightarrow$. Figure reproduced from Hellmuth et al. (2020).

Considering the evolution of gene families, for instance, vertex labels may be used to distinguish gene duplication events from speciation and horizontal gene transfer Fitch (2000). Edge labels, on the other hand, may be used to designate (rare) events that change properties of genes, genomes, and organisms Hellmuth et al. (2018a) or to distinguish different fates of offspring genes such as the horizontal transfer into another genomes Geiß et al. (2018). Distance-based phylogenetics can be seen as special case of the latter setting, where edges are weighted by evolutionary distances Semple and Steel (2003). Relations on $L$ are naturally defined as functions of the edge and/or vertex labels along the unique path connecting a pair of leaves. For instance, evolutionary distances are simply the sum of the edge length; the edge set of Pairwise Compatibility Graphs requires the path length (i.e., sum of edge-weights) to fall between given bounds Calamoneri and Sinaimeri (2016); a pair of genes are orthologs, a key relation in functional genomics, if their last common ancestor $\operatorname{lca}_{T}(x, y)$ is labeled as speciation; a directed xenology relation is defined by asking whether there is a "transfer edge" on the path between $\operatorname{lca}_{T}(x, y)$ and $y$.

In all these examples the mathematical interest is in the inverse problem. Given a relation or a set of relations and a rule relating labeled trees to the relation(s), one asks (i) when does a tree $T$ exist that explains the given relation, (ii) is there a unique explaining tree $T$ that is minimal in some sense (usually edge contraction), and (iii) can a (minimal) explaining tree be constructed efficiently from the given data. For the vertex-labeled case, symbolic ultrametrics Böcker and Dress (1998) and 2-structures Ehrenfeucht and Rozenberg (1990); Hellmuth et al. (2017) provide a comprehensive answer. Edge labels also have been studied extensively. For distances, the 4-point condition Buneman (1971) characterize the "additive" metrics deriving from trees. For rare events, where $x \sim y$ if they are separated by exactly one event, a complete characterization was provided in Hellmuth et al. (2018a). For PCGs (which exclude the possibility of no event along an edge), on the other hand, only partial results are known Calamoneri and Sinaimeri (2016).

In this contribution we are interested in a generalization of Fitch relations. These relations were introduced to model so-called horizontal gene transfer (HGT) and formalized in Geiß et al. (2018); Hellmuth 
et al. (2018b) based on Walter M. Fitch's definition of xenology Fitch (2000). Two genes $x$ and $y$ are in the directed Fitch relation $(x \rightarrow y)$ if there is at least one event (e.g. HGT) on the path between lca $(x, y)$ and $y$ Geiß et al. (2018) and they are in the symmetrized (or undirected) Fitch relation $(x \sim y)$ if there is at least one event on the path between $x$ and $y$ Hellmuth and Seemann (2019). While directed Fitch relations "implicitly" contain information about the directions of edges in a rooted tree, this information is lost in symmetrized Fitch relations. In particular, every symmetrized Fitch relation can be explained by a rooted tree if and only if it can be explained by an unrooted tree. Fig. 1 illustrates these concepts. Modeling different types of events by different labels yields a collection of (possibly non-disjoint) Fitch relations or, equivalently, multi-edge-colored graphs Hellmuth (2019) that can be used e.g. to distinguish genomic locations where the horizontally transferred gene copy is inserted, and adds to the information that is already provided by a single Fitch relation.

The directed Fitch relations corresponds to a certain subclass of directed cographs, which are explained by unique least-resolved trees Geiß et al. (2018); Hellmuth and Seemann (2019). The latter construction was further generalized to Fitch maps, or, equivalently, sets of Fitch relations, for every value of the label set. This imposes additional constraints beyond the obvious fact that one must have a Fitch relation for each label; again there is a unique least-resolved tree for every Fitch map Hellmuth et al. (2020). Symmetrized Fitch relations are of particular interest, as in practice, they can - to a large extent - be directly inferred from sequence similarities (best matches) on genomic data Schaller et al. (2020). The symmetrized Fitch relation coincide with the complete multi-partite graphs Hellmuth et al. (2018b). This begs the question whether symmetrized Fitch maps can be understood as simple superpositions of complete multi-partite graphs.

Our objects of interest - the Fitch relations and Fitch maps - are named in honor of Walter M. Fitch (1929-2011) due to his seminal work on xenology. To the best of our knowledge, they bear no relationship to Fitch's algorithm Fitch (1971), which - invented by the same W.M. Fitch - solves the "small parsimony problem", a combinatorial optimization problem concerned with minimizing the the total number differences between adjacent vertex labels (character states) in given phylogenetic trees. Since Fitch maps ask for the existence of an edge color rather then the number of edges carrying a given color, there is no apparent relation to a parsimony criterion for events (i.e., colors) on the edges.

Symmetric Fitch maps are distantly related to perfect phylogenies. In this important model of mathematical phylogenetics, each type of event (change of the state of a character) occurs exactly once, see e.g. Steel (1992b); Kannan and Warnow (1997); Fernández-Baca (2001). A perfect phylogeny is illustrated by the insertion of repetitive elements at a certain loci, from which they cannot be lost. In Fitch maps, each color may appear multiple times. Different edges of the same color may then be interpreted as insertions of members of a given repeat family at different loci. Different colors would then correspond to different repeat families. In the classical setting of phylogenetics the character states (i.e., presence or absence at each locus) are known for all leaves of the phylogenetic tree. A symmetric Fitch map, however, withholds the detailed information on the number and states of the characters, and instead only provides coarsegrained information on differences between sets of characters as observables. The recognition problem for Fitch maps therefore asks whether there exists a perfect phylogeny with an unknown number of binary characters for each color, that "explains" the available information.

The main result of this contribution states that a collection of binary relations is a symmetrized Fitch map if and only if each of them is a complete multi-partite graph and a certain set of subsplits defined by so-called complementary neighborhoods is compatible. This characterization has important consequence on the computational complexity. While symmetrized Fitch relations (or equivalently, undirected Fitch 
graphs) as well as directed Fitch maps can be recognized in polynomial time, this is no longer the case for symmetrized Fitch map; we show that their recognition problem is NP-complete. The restriction to maps where each pair of leaves $(x, y)$ has at most one label, however, remains polynomial. In particular, this work complements the results established in Hellmuth (2019); Hellmuth et al. (2020).

\section{Preliminaries}

Basic Notation For a finite set $X$ we write $[X \times X]_{\text {irr }}:=X \times X \backslash\{(x, x): x \in X\}$, and $\left(\begin{array}{c}X \\ k\end{array}\right):=\left\{X^{\prime} \subseteq\right.$ $\left.X:\left|X^{\prime}\right|=k\right\}$. The set $\mathcal{P}(X)$ denotes the power set of $X$. A partition of $X$ is a collection of pairwise disjoint non-empty sets $X_{1}, \ldots, X_{k}$ with $k \geq 1$ such that $X=X_{1} \bullet \ldots \bullet X_{k}$.

We consider undirected graphs $G=(V, E)$ with finite vertex set $V(G)=V$ and edge set $E(G)=$ $E \subseteq\left(\begin{array}{c}V \\ 2\end{array}\right)$, i.e., without loops and multiple edges. The complete graph $K_{|V|}$ has vertex set $V$ and edge set $E=\left(\begin{array}{c}V \\ 2\end{array}\right)$. Hence, $K_{1}$ denotes the single vertex graph and $K_{2}$ consist of two vertices and the connecting edge. The vertex degree $\operatorname{deg}_{G}(v)$ of $v \in V$ is the number of its adjacent vertices. A graph $H=(W, F)$ is a subgraph of $G=(V, E)$, denoted by $H \subseteq G$, if $W \subseteq V$ and $F \subseteq E$.

A subset $W \subseteq V$ is an independent set of $G=(V, E)$, if $\{x, y\} \notin E$ for all $x, y \in W . G=(V, E)$ is a complete multi-partite graph if there is a partition $V_{1}, \ldots, V_{k}, k \geq 1$ of $V$ such that $\{x, y\} \in E$ if and only if $x \in V_{i}$ and $y \in V_{j}$ with $i \neq j$. Thus, each part $V_{i}$ is a maximal independent set. A graph $G$ is a complete multi-partite graph if and only if it does not contain $K_{1}+K_{2}$, the disjoint union of $K_{1}$ and $K_{2}$, i.e., the graph with three vertices and a single edge as an induced subgraph, see e.g. Zverovich (1999).

Trees An (unrooted) tree $T=(V, E)$ is a connected, cycle-free graph. In a tree, there is a unique path $P_{T}(v, w)$ connecting any two vertices $v, w \in V(T)$. A vertex $v \in V(T)$ with $\operatorname{deg}_{T}(v)=1$ is a leaf, otherwise it is an inner vertex. The set of inner vertices is denoted by ${ }^{\circ}(T)$. Analogously, an edge $e=\{v, w\} \in E(T)$ with $v, w \in \stackrel{\circ}{V}(T)$ is an inner edge, and an outer edge, otherwise. The set of inner edges of a tree $T$ is denoted by $\stackrel{\circ}{E}(T)$. The tree $T$ is binary if $\operatorname{deg}_{T}(v)=3$ for every $v \in V(T) \backslash \mathcal{L}(T)$. An (unrooted) tree $T$ is phylogenetic if $\operatorname{deg}_{T}(v) \geq 3$ for every vertex $v \in V(T) \backslash \mathcal{L}(T)$. A star tree is a tree that has exactly one inner vertex and at least two leaves. Moreover, we say a tree $T$ is less resolved than a tree $T^{\prime}$, denoted by $T<T^{\prime}$, if $T$ can be obtained from $T^{\prime}$ by a non-empty sequence of edge-contractions.

Remark. From here on we consider only phylogenetic trees, and refer to them simply as trees.

Subsplits and Quartets A subsplit $A \mid B$ on a set $X$ is an unordered pair of two disjoint and non-empty subsets $A, B \subseteq X$, i.e. $A|B=B| A$. A subsplit $A \mid B$ is trivial if $\min \{|A|,|B|\}=1$, and it is a quartet if $|A|=|B|=2$. In the latter case we write $a b \mid c d$ instead of $\{a, b\} \mid\{c, d\}$. A subsplit $A \mid B$ on $X$ is a split on $X$ if $A \cup B=X$. A subsplit $A \mid B$ on $X$ is displayed by a tree $T$ with $\mathcal{L}(T)=X$ if there is an edge $e \in E(T)$ such that $A \subseteq \mathcal{L}\left(T_{1}\right)$ and $B \subseteq \mathcal{L}\left(T_{2}\right)$, where $T_{1}$ and $T_{2}$ are the connected components of $T \backslash e:=(V(T), E(T) \backslash\{e\})$. In this case we call $e$ a splitting edge w.r.t. $A \mid B$. Clearly, removal of an edge in $T$ yields always a split $\mathcal{L}\left(T_{1}\right) \mid \mathcal{L}\left(T_{2}\right)$ that is displayed by $T$. Hence, a subsplit $A \mid B$ is displayed by $T$ if there is a split $A^{\prime} \mid B^{\prime}$ in $T$ with $A \subseteq A^{\prime}$ and $B \subseteq B^{\prime}$. A set $\mathcal{S}$ of subsplits is called compatible if there is a tree $T$ that displays every subsplit in $\mathcal{S}$. The set $\mathcal{S}(T)$ comprises all splits on $X$ displayed by $T$ and the set $\mathcal{Q}(T)$ comprises all quartets that are displayed by $T$.

The relation between trees and split systems is captured by the following well-known result Buneman (1971), see (Semple and Steel, 2003, Section 3.1) for a detailed discussion. In the setting of $X$-trees, the taxa $X$ are mapped to vertices of the $T$ with degree at most 2 by a not necessarily injective map 
$p: X \rightarrow V(T)$. Since in our setting there is a one-to-one correspondence of $X$ and the leaves of $T$, i.e., $p$ is injective, the split system $\mathcal{S}$ necessarily contains all trivial splits $\{x\} \mid X \backslash\{x\}$ with $x \in X$.

Proposition 2.1 (Splits-Equivalence Theorem). Let $\mathcal{S}$ be a collection of splits on $X$ that contains all trivial splits. Then, there is a tree $T$ with leaf set $X$ such that $\mathcal{S}=\mathcal{S}(T)$ if and only if for all pairs of distinct splits $A_{1}\left|B_{1}, A_{2}\right| B_{2} \in \mathcal{S}$ at least one of the four intersections $A_{1} \cap A_{2}, A_{1} \cap B_{2}, B_{1} \cap A_{2}$ and $B_{1} \cap B_{2}$ is empty. Moreover, if such a tree exists, then $T$ is unique up to isomorphism.

For later reference we state a simple consequence of Proposition 2.1 .

Corollary 2.2. Let $\mathcal{S}$ be a collection of subsplits on $X$. If there are two subsplits $A_{1} \mid A_{2}$ and $B_{1} \mid B_{2}$ in $\mathcal{S}$ such that all four intersections $A_{1} \cap B_{1}, A_{1} \cap B_{2}, A_{2} \cap B_{1}$ and $A_{2} \cap B_{2}$ are non-empty, then $\mathcal{S}$ is not compatible.

Proof: Let $\mathcal{S}$ be a collection of subsplits on $X$, and suppose that are two subsplits $A_{1} \mid B_{1}$ and $A_{2} \mid B_{2}$ in $\mathcal{S}$ such that none of the sets $A_{1} \cap A_{2}, A_{1} \cap B_{2}, B_{1} \cap A_{2}$ and $B_{1} \cap B_{2}$ is empty. Assume for contradiction that $\mathcal{S}$ is compatible, i.e., there is a tree $T$ that displays $S$. Thus, there is a split $A_{1}^{\prime} \mid A_{2}^{\prime}$ and a split $B_{1}^{\prime} \mid B_{2}^{\prime}$ in $T$ such that $A_{1} \subseteq A_{1}^{\prime}, A_{2} \subseteq A_{2}^{\prime}, B_{1} \subseteq B_{1}^{\prime}$ and $B_{2} \subseteq B_{2}^{\prime}$. However, by assumption all four intersections $A_{i}^{\prime} \cap B_{j}^{\prime} \supseteq A_{i} \cap B_{j} \neq \emptyset$ with $i, j \in\{1,2\}$, and hence, by Proposition 2.1, such a tree $T$ cannot exist. Therefore, $\mathcal{S}$ is not compatible.

\section{Symmetrized Fitch maps}

Definition 3.1. Let $M$ be an arbitrary finite set of colors. An edge-labeled tree $(T, \lambda)$ on $X$ (with $M$ ) is a tree $T=(V, E)$ with $\mathcal{L}(T)=X$ together with a map $\lambda: E \rightarrow \mathcal{P}(M)$.

We will often refer to the map $\lambda$ as the edge-labeling and call $e$ an $m$-edge if $m \in \lambda(e)$, and an $\emptyset$-edge if $\lambda(e)=\emptyset$. Note that the choice of $m \in \lambda(e)$ may not be unique and an edge can be both, an $m$ - and an $m^{\prime}$-edge at the same time.

Definition 3.2. A map $\varepsilon:[X \times X]_{\text {irr }} \rightarrow \mathcal{P}(M)$, where $X$ is a non-empty set of "leaves" and $M$ is a non-empty set of "colors", is a symmetrized Fitch map if there is an edge-labeled tree $(T, \lambda)$ with leaf set $X$ and edge labeling $\lambda: E(T) \rightarrow \mathcal{P}(M)$ such that for every pair $(x, y) \in[X \times X]_{\text {irr }}$ it holds that

$$
m \in \varepsilon(x, y) \Longleftrightarrow \text { there is an } m \text {-edge on the path from } x \text { to } y \text {. }
$$

In this case we say that $\varepsilon:[X \times X]_{\text {irr }} \rightarrow \mathcal{P}(M)$ explains $(T, \lambda)$.

Every symmetrized Fitch map is symmetric, i.e., $\varepsilon(x, y)=\varepsilon(y, x)$ for every distinct $x, y \in X$. Furthermore, every symmetric map $\varepsilon:[X \times X]_{\text {irr }} \rightarrow \mathcal{P}(M)$ with $|X|=2$ is a symmetrized Fitch map.

Remark. From here on we assume w.l.o.g. that $\varepsilon$ is symmetric and $|X| \geq 3$.

Figure 2 provides an illustrative example of a symmetrized Fitch map $\varepsilon:[X \times X]_{\text {irr }} \rightarrow \mathcal{P}(M)$ and one of its corresponding edge-labeled trees $(T, \lambda)$. In particular, Figure 3 shows that the corresponding edge-labeled trees for $\varepsilon$ may not be unique in general. Every map $\varepsilon:[X \times X]_{\text {irr }} \rightarrow \mathcal{P}(M)$ can also be interpreted as a set of $|M|$ not necessarily disjoint binary relations (or equivalently graphs) on $X$ defined by the sets $\left\{(x, y) \in[X \times X]_{\text {irr }}: m \in \varepsilon(x, y)\right\}$ of pairs (or equivalently undirected edges) for every fixed color $m \in M$. 
a)

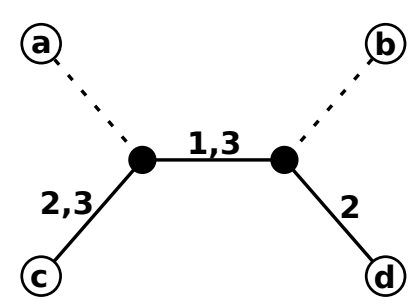

b)

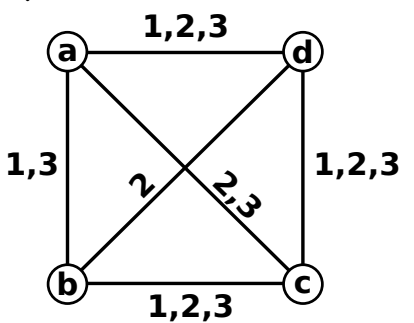

Fig. 2: The edge-labeled tree $(T, \lambda)$ with leaf set $\mathcal{L}(T)=\{a, b, c, d\}=: X$ on the left-hand side explains the symmetrized Fitch map $\varepsilon:[X \times X]_{\text {irr }} \rightarrow \mathcal{P}(M)$ with the color set $M=\{1,2,3\}$ on the right-hand side. Dashedlined edges $e$ in $T$ have label $\lambda(e)=\emptyset$. Moreover, an edge $\{x, y\}$ in the the right-hand side graph has label $i$ if and only if $i \in \varepsilon(x, y)$. By definition, symmetrized Fitch maps are symmetric, i.e., $m \in \varepsilon(x, y)$ if and only if $m \in \varepsilon(y, x)$ for all $m \in M$. However, symmetrized Fitch maps are not transitive in general. To see this, observe that $1 \in \varepsilon(a, b)$ and $1 \in \varepsilon(b, c)$ but $1 \notin \varepsilon(a, c)$.

Definition 3.3. The graph-representation of a map $\varepsilon:[X \times X]_{\text {irr }} \rightarrow \mathcal{P}(M)$ w.r.t. a color $m \in M$ is the (undirected) graph $\mathcal{G}_{m}(\varepsilon)$ with the vertex set $V\left(\mathcal{G}_{m}(\varepsilon)\right):=X$ and the edge set $E\left(\mathcal{G}_{m}(\varepsilon)\right):=$ $\left\{\{x, y\} \in\left(\begin{array}{c}X \\ 2\end{array}\right): m \in \varepsilon(x, y)\right\}$.

Following the approach by Hellmuth et al. (2020), we start by considering neighborhoods in this graph representation.

Definition 3.4 ((Hellmuth et al., 2020, Def. 3.3)). The (complementary) neighborhood of vertex $y \in X$ and a given color $m \in M$ w.r.t. $\varepsilon:[X \times X]_{\text {irr }} \rightarrow \mathcal{P}(M)$ is the set

$$
N_{\neg m}[y]:=\{x \in X \backslash\{y\}: m \notin \varepsilon(x, y)\} \cup\{y\}
$$

We write $\mathcal{N}_{\neg m}[\varepsilon]:=\left\{N_{\neg m}[y]: y \in X\right\}$ for the set of complementary neighborhoods of $\varepsilon$ and a particular color $m \in M$. Note that there might be distinct leaves $y, y^{\prime} \in X$ or distinct colors $m, m^{\prime} \in M$ such that $N_{\neg m}[y]=N_{\neg m^{\prime}}\left[y^{\prime}\right]$. Moreover, we emphasize that $\mathcal{N}_{\neg m}[\varepsilon]$ is not a multi-set, i.e. if $N_{\neg m}[y]=$ $N_{\neg m^{\prime}}\left[y^{\prime}\right]$ but $y \neq y^{\prime}$ or $m \neq m^{\prime}$, then they only contribute once to $\mathcal{N}_{\neg m}[\varepsilon]$.

\subsection{Characterization of monochromatic symmetrized Fitch maps}

A map $\varepsilon:[X \times X]_{\text {irr }} \rightarrow \mathcal{P}(M)$ is monochromatic if $\varepsilon(x, y)=\{m\}$ or $\varepsilon(x, y)=\emptyset$ for all distinct $x, y \in X$ and some fixed color $m \in M$. Hence, for monochromatic maps we can assume w.l.o.g. that $|M|=1$. Monochromatic symmetrized Fitch maps are equivalent to the "undirected Fitch graphs" studied by Hellmuth et al. (2018b).

For later reference we briefly recall some key results for this special case.

Lemma 3.5 ((Hellmuth et al. 2018b, Lemma $0.3 \&$ Thm. 0.5)). Let $\varepsilon:[X \times X]_{\text {irr }} \rightarrow \mathcal{P}(M)$ be a monochromatic map with $M=\{m\}$. Then, the following statements are equivalent:

1. $\varepsilon$ is a (monochromatic) symmetrized Fitch map.

2. $\mathcal{G}_{m}(\varepsilon)$ does not contain a $K_{1}+K_{2}$ as an induced subgraph. 
a)

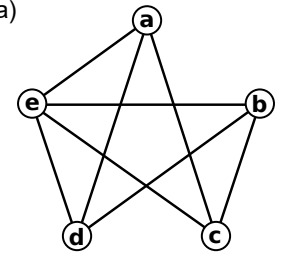

b)

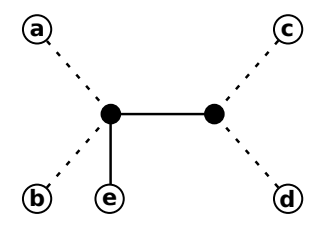

c)

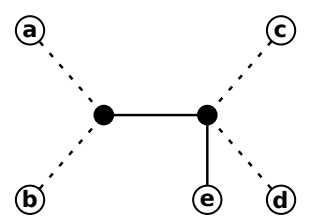

Fig. 3: Let $\varepsilon:[X \times X]_{\text {irr }} \rightarrow \mathcal{P}(M)$ be a symmetric map with $X:=\{a, b, c, d, e\}$ and $M:=\{m\}$, where for every distinct $x, y \in X$ we have $m \in \varepsilon(x, y)$ if and only if the shown graph in Panel a) contains the edge $\{x, y\}$. Moreover, there are two edge-labeled trees shown in Panel b) and c), where solid lines and dashed lines represent the edge-label $\{m\}$ and $\emptyset$, respectively. We observe that both edge-labeled trees explains $\varepsilon$. Thus, $\varepsilon$ is a (monochromatic) symmetrized Fitch relation. For instance, $m \notin \varepsilon(a, b) \cup \varepsilon(c, d)$ but $m \in \varepsilon(a, c)$ imply that every edge-labeled tree, which explains $\varepsilon$, needs at least one inner edge. Thus, these two trees have the fewest numbers of vertices among all trees that may explain $\varepsilon$ and are known as so-called "minimally-resolved" trees. The latter arguments imply that minimally-resolved trees need not to be unique; a fact that has also been observed in Hellmuth et al. (2018b).

\section{3. $\mathcal{G}_{m}(\varepsilon)$ is a complete multi-partite graph.}

Using Lemma 3.5, we can derive the following alternative characterization:

Proposition 3.6. Let $\varepsilon:[X \times X]_{\text {irr }} \rightarrow \mathcal{P}(M)$ be a monochromatic map with $M=\{m\}$. Then, the following statements are equivalent:

1. $\varepsilon$ is a (monochromatic) symmetrized Fitch map.

2. For every three pairwise distinct $a, b, c \in X$ with $m \notin \varepsilon(a, b)$ and $m \notin \varepsilon(b, c)$, we have $m \notin$ $\varepsilon(a, c)$.

\section{3. $\mathcal{N}_{\neg m}[\varepsilon]$ is a partition of $X$.}

Proof: Let $\varepsilon:[X \times X]_{\text {irr }} \rightarrow \mathcal{P}(M)$ be a monochromatic map with $M=\{m\}$. In the following will make frequent use of the fact that $\varepsilon(a, b)=\varepsilon(b, a)$ and, therefore, $m \in \varepsilon(a, b)$ if and only if $\{a, b\} \in E\left(\mathcal{G}_{m}(\varepsilon)\right)$.

First, assume that Statement (1) is satisfied. Lemma 3.5 implies that $\mathcal{G}_{m}(\varepsilon)$ does not contain a $K_{1}+K_{2}$ as an induced subgraph. Hence, for arbitrary pairwise distinct $a, b, c \in X$ with $m \notin \varepsilon(a, b)$ and $m \notin$ $\varepsilon(b, c)$, it must hold that $m \notin \varepsilon(a, c)$. Thus, Statement (2) holds.

Now, assume that Statement (2) is satisfied. Recall that the set $\mathcal{N}_{\neg m}[\varepsilon]$ is a partition of $X$ if $\mathcal{N}_{\neg m}[\varepsilon]$ is a collection of pairwise disjoint non-empty sets $N_{1}, \ldots, N_{k}$ such that $X=N_{1} \bullet \ldots \cup N_{k}$. Since $y \in N_{\neg m}[y]$, we conclude that every neighborhood in $\mathcal{N}_{\neg m}[\varepsilon]$ is non-empty and that $\bigcup_{y \in X} N_{\neg m}[y]=$ $X$. To this end, let $y, y^{\prime} \in X$ be two distinct vertices that satisfy $N_{\neg m}[y] \cap N_{\neg m}\left[y^{\prime}\right] \neq \emptyset$. Thus, we must verify that $N_{\neg m}[y]=N_{\neg m}\left[y^{\prime}\right]$. Moreover, we can assume w.l.o.g. that $\left|N_{\neg m}[y]\right| \leq\left|N_{\neg m}\left[y^{\prime}\right]\right|$. Now, we continue to show that $m \notin \varepsilon\left(y, y^{\prime}\right)=\varepsilon\left(y^{\prime}, y\right)$. To this end, we assume for contradiction that $m \in \varepsilon\left(y, y^{\prime}\right)=\varepsilon\left(y^{\prime}, y\right)$. Therefore, $y \notin N_{\neg m}\left[y^{\prime}\right]$ and $y^{\prime} \notin N_{\neg m}[y]$. Thus, $y, y^{\prime} \notin N_{\neg m}[y] \cap N_{\neg m}\left[y^{\prime}\right]$. This, together with $N_{\neg m}[y] \cap N_{\neg m}\left[y^{\prime}\right] \neq \emptyset$, implies that there is a vertex $x \in N_{\neg m}[y] \cap N_{\neg m}\left[y^{\prime}\right]$ such that $x, y$ and $y^{\prime}$ are pairwise distinct. However, $m \notin \varepsilon(x, y)=\varepsilon(y, x)$ and $m \notin \varepsilon\left(x, y^{\prime}\right)$. In summary, we have $m \notin \varepsilon(y, x), m \notin \varepsilon\left(x, y^{\prime}\right)$ and $m \in \varepsilon\left(y, y^{\prime}\right)$; a contradiction to Statement (2). Thus, $m \notin \varepsilon\left(y, y^{\prime}\right)=\varepsilon\left(y^{\prime}, y\right)$. The latter implies that $\left\{y, y^{\prime}\right\} \subseteq N_{\neg m}[y]$. Now, let $x \in N_{\neg m}\left[y^{\prime}\right]$. If $x \in\left\{y, y^{\prime}\right\}$, 
then we have $x \in\left\{y, y^{\prime}\right\} \subseteq N_{\neg m}[y]$. Moreover, if $x \notin\left\{y, y^{\prime}\right\}$, then $x, y$ and $y^{\prime}$ are pairwise distinct. In this case, $m \notin \varepsilon\left(x, y^{\prime}\right)$ and $m \notin \varepsilon\left(y^{\prime}, y\right)$ together with Statement (2) implies that $m \notin \varepsilon(x, y)$. Therefore, $x \in N_{\neg m}[y]$. In either case, we have $x \in N_{\neg m}[y]$. Thus, $N_{\neg m}\left[y^{\prime}\right] \subseteq N_{\neg m}[y]$. This, together with $\left|N_{\neg m}[y]\right| \leq\left|N_{\neg m}\left[y^{\prime}\right]\right|$, implies that $N_{\neg m}[y]=N_{\neg m}\left[y^{\prime}\right]$. Therefore, Statement (3) is true.

Finally, we show that Statement (3) implies Statement (1). Using contraposition, we assume that $\varepsilon$ is not a symmetrized Fitch map. Then, we conclude by Lemma 3.5 that $\mathcal{G}_{m}(\varepsilon)$ contains an $K_{1}+K_{2}$ as an induced subgraph. Let $\mathcal{G}_{m}(\varepsilon)[\{a, b, c\}]$ be an induced subgraph that is isomorphic to $K_{1}+K_{2}$. We can assume w.l.o.g. that $m \notin \varepsilon(a, b), m \notin \varepsilon(a, c)$ and $m \in \varepsilon(b, c)$. The latter implies that $b \notin N_{\neg m}[c]$. This, together with $b \in N_{\neg m}[b]$, implies that $N_{\neg m}[b] \neq N_{\neg m}[c]$. Moreover, we have $a \in N_{\neg m}[b] \cap N_{\neg m}[c]$. Taken the latter arguments together, we observe that $\mathcal{N}_{\neg m}[\varepsilon]$ cannot be a partition of $X$. Thus, if Statement (3) is satisfied, then Statement (1) must be satisfied as well.

A natural special case is to consider maps $\varepsilon:[X \times X]_{\text {irr }} \rightarrow \mathcal{P}(M)$ that assign to each pair $(x, y)$ at most one label. In this case, $\varepsilon$ reduces to a map $\varepsilon:[X \times X]_{\mathrm{irr}} \rightarrow M \cup\{\emptyset\}$.

Proposition 3.7. The map $\varepsilon:[X \times X]_{\text {irr }} \rightarrow \mathcal{P}(M)$ is a symmetrized Fitch map that satisfies $|\varepsilon(x, y)| \leq 1$ for all distinct $x, y \in X$ if and only if $\varepsilon$ is a monochromatic symmetrized Fitch map.

Proof: Clearly, every monochromatic symmetrized Fitch map $\varepsilon$ is a symmetrized Fitch map with $|\varepsilon(x, y)| \leq 1$ for all distinct $x, y \in X$. Now, suppose that $\varepsilon:[X \times X]_{\text {irr }} \rightarrow \mathcal{P}(M)$ is a symmetrized Fitch map that satisfies $|\varepsilon(x, y)| \leq 1$. Then, assume for contradiction that $\varepsilon$ is not monochromatic. Thus, there are leaves $a, b, c, d \in X$ with $\varepsilon(a, b)=\{m\}$ and $\varepsilon(c, d)=\left\{m^{\prime}\right\}$ for distinct $m, m^{\prime} \in M$. Since $\varepsilon$ is a symmetrized Fitch map, there is an edge-labeled tree $(T, \lambda)$ that explains $\varepsilon$. The latter two arguments imply that $T$ contains an $m$-edge $e$ and $m^{\prime}$-edge $f$. Now, consider a vertex-maximal path $P$ in $T$ that contains $e$ and $f$. Clearly, $P$ must contain two leaves $x, y \in X$ as its end-vertices. But then $m, m^{\prime} \in \varepsilon(x, y)$ implies $|\varepsilon(x, y)|>1$; a contradiction.

Finally, we characterize least-resolved trees for a monochromatic symmetrized Fitch map $\varepsilon$ : An edgelabeled tree $\left(T^{*}, \lambda^{*}\right)$ is least-resolved for $\varepsilon$ if there is no tree $T<T^{*}$ and no labeling $\lambda$ such that $(T, \lambda)$ also explains $\varepsilon$. Such trees can be constructed using the fact that $\mathcal{G}_{m}(\varepsilon)$ is identified by the set $\mathcal{I}$ of its maximal independent sets since it is a complete multi-partite graph (cf. L. 3.5). As remarked above, we assume $|X| \geq 3$ to avoid trivial cases.

Definition 3.8. Let $\varepsilon:[X \times X]_{\text {irr }} \rightarrow \mathcal{P}(M)$ be a monochromatic symmetrized Fitch map with $M=\{m\}$ and $|X| \geq 3$ with graph-representation $\mathcal{G}_{m}(\varepsilon)$. Then, $\mathcal{T}_{\varepsilon}$ is the collection of all edge-labeled trees $(T, \lambda)$ on $X$ that satisfy the following properties:

1. If $\mathcal{I}_{2}:=\{I \in \mathcal{I}:|I| \geq 2\}=\emptyset$, i.e. if $\mathcal{G}_{m}(\varepsilon) \simeq K_{|X|}$, then $(T, \lambda)$ is the star tree on $X$, and all edges $e \in E(T)$ with one possible exception are $m$-edges.

2. If $\mathcal{I}_{2} \neq \emptyset$, then
(a) $|\stackrel{V}{V}(T)|=\left|\mathcal{I}_{2}\right|$;
(b) for each $I \in \mathcal{I}_{2}$ there is a unique inner vertex $v_{I} \in \stackrel{\circ}{V}(T)$ such that $\left\{x, v_{I}\right\} \in E(T)$ for every $x \in I$;
(c) every outer edge $\{x, v\} \in E(T)$ with $x \in \bigcup_{I \in \mathcal{I}_{2}} I$ is labeled by $\lambda(\{x, v\})=\emptyset$;
(d) every outer edge $\{x, v\} \in E(T)$ with $x \in X \backslash \bigcup_{I \in \mathcal{I}_{2}} I$ is labeled by $\lambda(\{x, v\})=\{m\}$; 

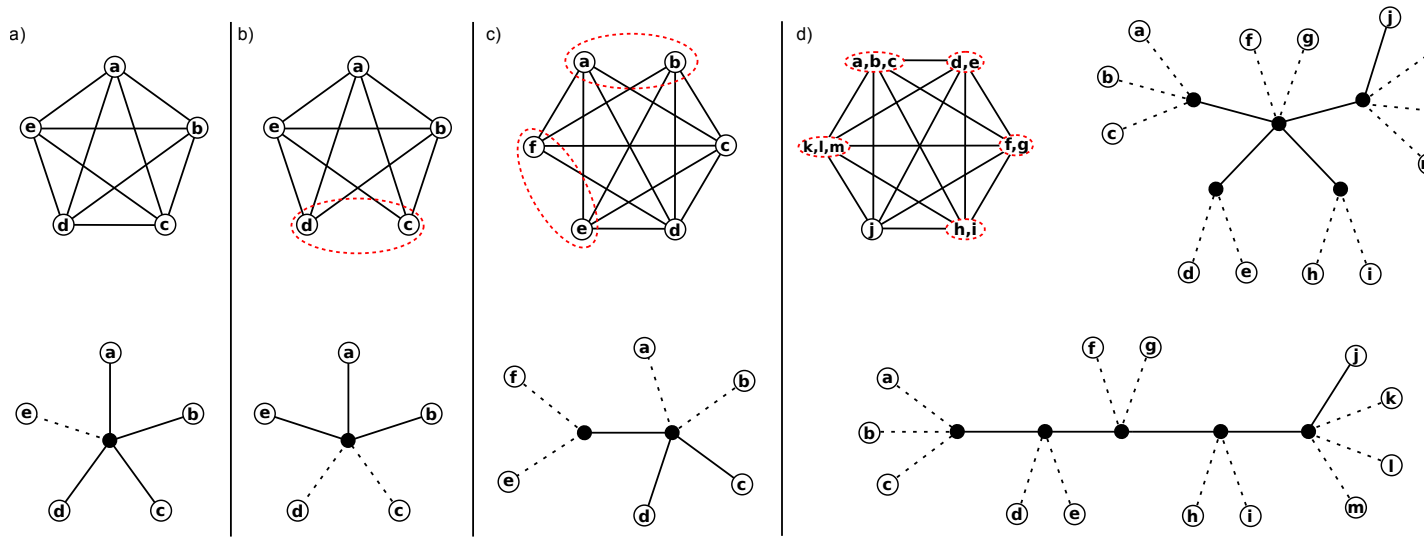

Fig. 4: Graph representations $\mathcal{G}_{m}(\varepsilon)$ (Fitch graphs) of several monochromatic symmetrized Fitch maps $\varepsilon$ : $[X \times$ $X]_{\text {irr }} \rightarrow \mathcal{P}(M), M=\{m\}$ (upper parts) together with edge-labeled trees $(T, \lambda)$ that explain $\varepsilon$ (drawn next to the respective $\mathcal{G}_{m}(\varepsilon)$ ). The Fitch graphs are complete bipartite graphs and thus, are identified by the set $\mathcal{I}$ of their maximal independent sets. All maximal independent sets of size at least two are highlighted by red dashed-lined ellipses. All trees satisfy the conditions in Def. 3.8 and are thus, by Prop. 3.9 least-resolved and, by Cor. 3.10 minimally-resolved for each $\varepsilon$.

(e) every inner edge $e \in \stackrel{\circ}{E}(T)$ is labeled by $\lambda(e)=\{m\}$.

Note that there are no restrictions on the arrangement of the inner edges in Def. 3.8 as long as $(T, \lambda)$ results in a phylogenetic tree. For instance, the inner edges could be arranged as a star-graph or as a path. See Fig. 4 for an illustrative example.

Proposition 3.9. Let $\varepsilon:[X \times X]_{\text {irr }} \rightarrow \mathcal{P}(M)$ be a monochromatic symmetrized Fitch map with $M=$ $\{m\}$, and let $\mathcal{T}_{\varepsilon}$ be the collection of trees as specified in Def. 3.8 Then, $\mathcal{T}_{\varepsilon}$ is the set of all least-resolved trees for $\varepsilon$.

Proof: Let $\varepsilon:[X \times X]_{\text {irr }} \rightarrow \mathcal{P}(M)$ be a monochromatic symmetrized Fitch map, and $\mathcal{I}, \mathcal{I}_{2}$ and $\mathcal{T}_{\varepsilon}$ as specified in Def. 3.8 First, assume that $\mathcal{I}_{2}=\emptyset$, i.e. $\mathcal{G}_{m}(\varepsilon) \simeq K_{|X|}$. It is an easy exercise to verify that all least-resolved trees for $\varepsilon$ must be a star tree, and all edges with one possible exception are $m$-edges. Hence, all such trees are, by Def. 3.8.11), contained in $T_{\varepsilon}$.

Let us now assume that $\mathcal{I}_{2} \neq \emptyset$ and let $\left(T^{*}, \lambda^{*}\right)$ be a least-resolved tree for $\varepsilon$. We show that $\left(T^{*}, \lambda^{*}\right) \in T_{\varepsilon}$. To this end, we assume first, for contradiction, that there is an inner edge $e \in \stackrel{\circ}{E}\left(T^{*}\right)$ with $\lambda(e)=\emptyset$, and consider the edge-labeled tree $\left(T^{\prime}, \lambda^{\prime}\right)$ obtained from $T^{*}$ by contraction of $e$ and keeping the remaining edge labels of $\lambda^{*}$. Note that $T^{\prime}$ is still a phylogenetic tree. Let $x, y \in X$ be two distinct leaves. If $\varepsilon(x, y)=\emptyset$, then the path $P_{T^{*}}(x, y)$ can have only $\emptyset$-edges. It is easy to see that this property is preserved by $\left(T^{\prime}, \lambda^{\prime}\right)$. If $\varepsilon(x, y)=\{m\}$, then the path $P_{T^{*}}(x, y)$ contains an $m$-edge. Since we only contracted the single inner $\emptyset$-edge to obtain $T^{\prime}$, the path $P_{T^{\prime}}(x, y)$ still contains this $m$-edge. Consequently, $\left(T^{\prime}, \lambda^{\prime}\right)$ explains $\varepsilon$ but $T^{\prime}<T^{*}$; contradicting the fact that $\left(T^{*}, \lambda^{*}\right)$ is least-resolved for $\varepsilon$. Hence, every inner edge $e \in \stackrel{\circ}{E}\left(T^{*}\right)$ is an $m$-edge, and thus, Statement (2e) holds.

To see that Statement (2c) is satisfied, observe that for every $x \in \bigcup_{I \in \mathcal{I}_{2}} I$ there is an $I \in \mathcal{I}_{2}$ with $x \in I$, and there is a $y \in I \backslash\{x\}$. Hence, $\varepsilon(x, y)=\emptyset$, and thus, the path $P_{T^{*}}(x, y)$ contains only edges 
$e$ with $\lambda^{*}(e)=\emptyset$. In particular, for the outer edge $\{x, v\} \in E\left(T^{*}\right)$, we therefore have $\lambda^{*}(\{x, v\})=\emptyset$. Hence, Statement (2c) holds.

To see that Statement (2b) is satisfied, we assume first, for contradiction, that there are two vertices $x, y \in I \in \mathcal{I}_{2}$ such that $\left\{x, v_{I}\right\} \in E\left(T^{*}\right)$ and $\left\{y, v_{I}^{\prime}\right\} \in E\left(T^{*}\right)$ but $v_{I} \neq v_{I}^{\prime}$. By Statement (2e), there is an inner $m$-edge contained in $P_{T^{*}}(x, y)$ since $v_{I}$ and $v_{I}^{\prime}$ are distinct inner vertices. Thus, $\varepsilon(x, y)=\{m\} \neq \emptyset$; a contradiction to $x, y \in I$. By similar arguments, $\{x, v\},\left\{x^{\prime}, v\right\} \in E\left(T^{*}\right)$ with $x \in I \in \mathcal{I}_{2}$ and $x^{\prime} \in I^{\prime} \in \mathcal{I}_{2}$ imply $x, y \in I \cap I^{\prime}$, and since $\mathcal{I}_{2}$ forms a partition, $I=I^{\prime}$. Thus, the required uniqueness in Statement (2b) holds. The previous arguments together, imply that Statement (2b) holds.

We continue by showing that every inner vertex $v \in \stackrel{\circ}{V}\left(T^{*}\right)$ is incident to an outer $\emptyset$-edge $\{v, x\} \in$ $E\left(T^{*}\right)$. If $T$ is a star graph, i.e. $v \in \stackrel{V}{ }\left(T^{*}\right)$ is the only inner vertex, then there must be an outer edge $\{v, x\} \in E\left(T^{*}\right)$ with $\lambda(\{v, x\})=\emptyset$, since $\mathcal{I}_{2} \neq \emptyset$. Now, assume that $T$ has inner edges. Moreover, assume for contradiction that there is an inner vertex $v \in \stackrel{\circ}{V}\left(T^{*}\right)$ such that every outer edge $\{v, x\} \in E\left(T^{*}\right)$ has label $\lambda^{*}(\{x, v\})=\{m\}$. Since $v \in \stackrel{\circ}{V}\left(T^{*}\right)$ and $T$ has inner edges, we can apply Statement (2e) to conclude that there is an inner $m$-edge $\{v, w\} \in \stackrel{\circ}{E}\left(T^{*}\right)$. Now, consider the edge-labeled tree $\left(T^{\prime}, \lambda^{\prime}\right)$ obtained from $T^{*}$ by contraction of $\{v, w\}$ and keeping the remaining edge labels of $\lambda^{*}$. Let $\varepsilon^{\prime}$ be the symmetrized Fitch relation explained by $\left(T^{\prime}, \lambda^{\prime}\right)$ and $x, y \in X$ be chosen arbitrarily. If $P_{T^{*}}(x, y)$ does not contain the $m$-edge $\{v, w\}$, then $P_{T^{\prime}}(x, y)=P_{T^{*}}(x, y)$ and the edge-labels along this path remain unchanged. Therefore, $\varepsilon^{\prime}(x, y)=\varepsilon(x, y)$. Now, suppose that $P_{T^{*}}(x, y)$ contains the edge $\{v, w\}$, and thus, $\varepsilon(x, y)=\{m\}$. If $P_{T^{\prime}}(x, y)$ does not contain any inner edge, then either $x$ or $y$ must be incident to $v$ in $T^{*}$, say $\{x, v\} \in E\left(T^{*}\right)$ and thus, $\lambda^{\prime}(\{x, v\})=\lambda^{*}(\{x, v\})=\{m\}$. Hence, $P_{T^{\prime}}(x, y)$ still contains an $m$-edge, and therefore, $\varepsilon^{\prime}(x, y)=\varepsilon(x, y)=\{m\}$. Otherwise, if $P_{T^{\prime}}(x, y)$ contains an inner edge, then it contains in particular an $m$-edge (cf. Statement (2e)). Again, $\varepsilon^{\prime}(x, y)=\varepsilon(x, y)=\{m\}$. Hence, we have shown that the symmetrized Fitch relation $\varepsilon^{\prime}$ that is explained by $\left(T^{\prime}, \lambda^{\prime}\right)$ and $\varepsilon$ are identical. Consequently, $\left(T^{*}, \lambda^{*}\right)$ is not a least-resolved tree for $\varepsilon$; a contradiction. Therefore, every inner vertex $v \in \stackrel{\circ}{V}\left(T^{*}\right)$ is incident to an outer $\emptyset$-edge $\{v, x\} \in E\left(T^{*}\right)$.

Now, let $\{x, v\} \in E\left(T^{*}\right)$ be an outer edge with $x \in X \backslash \bigcup_{I \in \mathcal{I}_{2}} I$. In particular, $x \in X \backslash \bigcup_{I \in \mathcal{I}_{2}} I$ implies that $\varepsilon(x, y)=\{m\}$ for every $y \in X \backslash\{x\}$. Assume, for contradiction, that $\lambda^{*}(\{x, v\})=\emptyset$. Due to the choice of $x$, every possible outer edge $\{y, v\} \in E\left(T^{*}\right)$ with $x \neq y$ must have label $\lambda^{*}(\{y, v\})=\{m\}$. Let us keep all edge-labels in $T^{*}$ except for $\{x, v\}$, which is relabeled to an $m$-edge. This, results in an edge-labeled tree $\left(T^{*}, \lambda^{\prime}\right)$ where $v$ is incident to $m$-edges only that still explains $\varepsilon$. But then, $\left(T^{*}, \lambda^{\prime}\right)$ and thus, $\left(T^{*}, \lambda^{*}\right)$ cannot be least-resolved, since for every inner vertex $v \in \stackrel{V}{ }\left(T^{*}\right)$ there must be an outer $\emptyset$-edge. Hence, Statement (2d) holds.

It remains to show that Statement (2a) is satisfied. Statement (2b) implies $|V(T)| \geq\left|\mathcal{I}_{2}\right|$. Assume, for contradiction, that $|\stackrel{\circ}{V}(T)|>\left|\mathcal{I}_{2}\right|$. Statement $(2 \mathrm{~b})$ and $|\stackrel{\circ}{V}(T)|>\left|\mathcal{I}_{2}\right|$ implies that there is an inner vertex $v \in \stackrel{\circ}{V}\left(T^{*}\right)$ that is not adjacent to a leaf $x \in I \in \mathcal{I}_{2}$. Hence, for every outer edge $\{v, x\} \in E\left(T^{*}\right)$ we have $x \in X \backslash \bigcup_{I \in \mathcal{I}_{2}} I$, and by Statement (2d), $\{v, x\}$ is an $m$-edge. Hence, $v \in V\left(T^{*}\right)$ is not incident to an outer $\emptyset$-edge $\{v, x\} \in E\left(T^{*}\right)$; a contradiction. Therefore, $\left|{ }^{\circ}(T)\right|=\left|\mathcal{I}_{2}\right|$, i.e., Statement (2a) is satisfied.

In summary, every least-resolved $\left(T^{*}, \lambda^{*}\right)$ satisfies either Statement (1) or (2a-e) and thus, $\left(T^{*}, \lambda^{*}\right) \in$ $\mathcal{T}_{\varepsilon}$. In particular, all least-resolved trees must have the same number of inner vertices, that is, one inner vertex (in case of Statement (1)) or $\left|\mathcal{I}_{2}\right|$ inner vertices (in case Statement (2)). Consequently, all leastresolved trees have the same number of vertices, that is, $|X|+1$ (Statement (1)) or $|X|+\left|\mathcal{I}_{2}\right|$ (Statement (2)).

It remains to show that every tree in $\mathcal{T}_{\varepsilon}$ is also least-resolved for $\varepsilon$. Hence, let $(T, \lambda) \in \mathcal{T}_{\varepsilon}$ be an edge- 
labeled tree. We show first that $(T, \lambda)$ explains $\varepsilon$. To this end, let $x, y \in X$ be distinct. If $\varepsilon(x, y)=\emptyset$, then $x, y$ are contained in the same independent set $I$ and, by construction (2b), the path $P_{T}(x, y)$ contains only the two outer $\emptyset$-edges $\left\{v_{I}, x\right\}$ and $\left\{v_{I}, y\right\}$. If $\varepsilon(x, y)=\{m\}$, then $x$ and $y$ are contained in distinct independent sets $I, I^{\prime} \in \mathcal{I}$, say $x \in I$ and $y \in I^{\prime}$. If $|I|=1$ then, by construction (2c), there is an $m$-edge $\{v, x\}$ for some $v \in \stackrel{\circ}{V}(T)$. Similarly, if $\left|I^{\prime}\right|=1$, there is an $m$-edge $\{v, y\}$. If $|I|>1$ and $\left|I^{\prime}\right|>1$, then Statement (2b) implies that $\{x, v\},\left\{y, v^{\prime}\right\} \in E(T)$ with $v \neq v^{\prime}$. Hence, the path $P_{T}(x, y)$ contains an inner edge $e \in \stackrel{\circ}{E}(T)$, which has by Statement (2e) the label $\lambda(e)=\{m\}$. Hence, in all cases for $\varepsilon(x, y)=\{m\}$, the path $P_{T}(x, y)$ contains an $m$-edge. In summary, $(T, \lambda)$ explains $\varepsilon$.

It remains to show that $(T, \lambda)$ is least-resolved for $\varepsilon$. If $(T, \lambda)$ is not least-resolved for $\varepsilon$ then there is a least-resolved tree $\left(T^{\prime}, \lambda^{\prime}\right)$ for $\varepsilon$ such that $T^{\prime}<T$ and therefore $\left|V\left(T^{\prime}\right)\right|<|V(T)|$. As argued above, if $\left(T^{*}, \lambda^{*}\right)$ is an arbitrary least-resolved tree for $\varepsilon$ we have by construction $\left|V\left(T^{*}\right)\right|=|V(T)|$. Therefore $\left|V\left(T^{\prime}\right)\right|<\left|V\left(T^{*}\right)\right|$, contradicting the fact that all least-resolved trees for $\varepsilon$ must have the same number of vertices. Consequently, $(T, \lambda)$ is least-resolved for $\varepsilon$.

In particular, Prop. 3.9 implies the following

Corollary 3.10. Let $\varepsilon$ be a monochromatic symmetrized Fitch map. Then, the least-resolved trees for $\varepsilon$ have the same number of vertices and, thus, in particular, the minimum number of vertices among all trees that explain $\varepsilon$, i.e., they are minimally-resolved trees for $\varepsilon$.

Corollary 3.11. Every monochromatic symmetrized Fitch map can be explained by an edge labeled tree $(T, \lambda)$ of diameter $\operatorname{diam}(T) \leq 4$, i.e., the length of each path in $T$ is four or less.

Proof: If $\left|\mathcal{I}_{2}\right| \leq 1$ and $(T, \lambda) \in \mathcal{T}_{\varepsilon}$, then, by construction, $\operatorname{diam}(T)=2$. Otherwise, if $\left|\mathcal{I}_{2}\right|>1$, then there is a tree $(T, \lambda) \in \mathcal{T}_{\varepsilon}$ such that all inner edges of $T$ share a common vertex as illustrated in Fig. 4 In this case, $\operatorname{diam}(T)=3$ if $\left|\mathcal{I}_{2}\right|=2$ and $\operatorname{diam}(T)=4$ if $\left|\mathcal{I}_{2}\right|>2$.

Corollary 3.11 can also be obtained from the explicit construction of rooted trees that explain undirected Fitch graphs Hellmuth et al. (2018b).

\subsection{Characterization of symmetrized Fitch maps}

Unfortunately, the properties in Prop. 3.6 are not sufficient to characterize non-monochromatic Fitch maps. To see this, consider the symmetric map $\varepsilon$ shown in Fig. 5. Then, we have $N_{\neg 1}[a]=N_{\neg 1}[c]=\{a, c\}$, $N_{\neg 1}[b]=N_{\neg 1}[d]=\{b, d\}, N_{\neg 2}[a]=N_{\neg 2}[b]=\{a, b\}$, and $N_{\neg 2}[c]=N_{\neg 2}[d]=\{c, d\}$. Hence, both $\mathcal{N}_{\neg 1}[\varepsilon]=\{\{a, c\},\{b, d\}\}$ and $\mathcal{N}_{\neg 2}[\varepsilon]=\{\{a, b\},\{c, d\}\}$ are partitions of $X=\{a, b, c, d\}$. As we shall prove in Lemma 3.16 below, every tree that explains $\varepsilon$ must display the quartets $a b \mid c d$ and $a c \mid b d$. However, by Corollary 2.2 the set $\{a b|c d, a c| b d\}$ of quartets is not compatible. Therefore, $\varepsilon$ cannot be a Fitch map.

Before we provide a characterization of symmetrized Fitch maps, we derive some necessary conditions.

Lemma 3.12. Let $\varepsilon:[X \times X]_{\text {irr }} \rightarrow \mathcal{P}(M)$ be a symmetrized Fitch map, and let $X^{\prime} \subseteq X$ and $M^{\prime} \subseteq M$. Then, the map $\varepsilon^{\prime}:\left[X^{\prime} \times X^{\prime}\right]_{\text {irr }} \rightarrow \mathcal{P}\left(M^{\prime}\right)$ with $\varepsilon^{\prime}(x, y):=\varepsilon(x, y) \cap M^{\prime}$ for every $(\bar{x}, y) \in\left[X^{\prime} \times \bar{X}^{\prime}\right]_{\text {irr }}$ is again a symmetrized Fitch map.

Proof: Let $\varepsilon:[X \times X]_{\text {irr }} \rightarrow \mathcal{P}(M)$ be a symmetrized Fitch map, and let $X^{\prime} \subseteq X$ and $M^{\prime} \subseteq M$. Let $\varepsilon^{\prime}:\left[X^{\prime} \times X^{\prime}\right]_{\text {irr }} \rightarrow \mathcal{P}\left(M^{\prime}\right)$ with $\varepsilon^{\prime}(x, y):=\varepsilon(x, y) \cap M^{\prime}$ for every $(x, y) \in\left[X^{\prime} \times X^{\prime}\right]_{\text {irr }}$ be a map.

Since $\varepsilon:[X \times X]_{\text {irr }} \rightarrow \mathcal{P}(M)$ is a symmetrized Fitch map, there is an edge-labeled tree $(T, \lambda)$ that explains $\varepsilon$. Now, create a tree $T^{\prime}$ from $T$, where every leaf $x \in X \backslash X^{\prime}$ in $T$ is deleted, and create an 
edge-labeling $\lambda^{\prime}: E\left(T^{\prime}\right) \rightarrow \mathcal{P}\left(M^{\prime}\right)$ with $\lambda^{\prime}(e):=\lambda(e) \cap M^{\prime}$ for every $e \in E\left(T^{\prime}\right)$. By construction, $m \in \varepsilon^{\prime}(x, y)$ if and only if the unique path between $x$ and $y$ in $T^{\prime}$ contains an $m$-edge for all $m \in M^{\prime}$ and $x, y \in X^{\prime}$. However, the tree $T^{\prime}$ might have vertices of degree 2 , and hence may not be a phylogenetic tree. However, we can further modify $T^{\prime}$ as follows: Suppose that there is a vertex $v$ of degree 2. Thus, there are two edges $e_{1}=\{v, w\}$ and $e_{2}=\{v, u\}$ in $T^{\prime}$. Now, we remove vertex $v$ and the two edges $e_{1}$ and $e_{2}$ from $T^{\prime}$ and add the edge $f=\{u, w\}$, and call the resulting tree $T^{\prime \prime}$. By construction, every path in $T^{\prime}$ between two leaves $x, y \in X^{\prime}$ that contains the edge $e_{1}$ or $e_{2}$ must now contain the edge $f$ in $T^{\prime \prime}$. We construct the edge-labeling $\lambda^{\prime \prime}: E\left(T^{\prime \prime}\right) \rightarrow \mathcal{P}\left(M^{\prime}\right)$ with $\lambda^{\prime \prime}(e):=\lambda^{\prime}(e)$ for all $e \in E\left(T^{\prime \prime}\right) \backslash f$ and $\lambda^{\prime \prime}(f):=\lambda^{\prime}\left(e_{1}\right) \cup \lambda^{\prime}\left(e_{2}\right)$. Then, for every $m \in M^{\prime}$ and every distinct $x, y \in X^{\prime}$, we have $m \in \varepsilon^{\prime}(x, y)$ if and only if $m \in \lambda^{\prime \prime}(e)$ for some edge $e \in P_{T^{\prime \prime}}(x, y)$. Clearly, $T^{\prime \prime}$ and $\lambda^{\prime \prime}$ can be iteratively modified as described above until no vertices with degree 2 remain, and hence we end up with an edge-labeled tree $(\tilde{T}, \tilde{\lambda})$. Thus, by construction of $\tilde{T}$ and $\tilde{\lambda}$, we have $m \in \varepsilon^{\prime}(x, y)$ if and only if the unique path between $x$ and $y$ in $\tilde{T}$ contains an $m$-edge for all $m \in M^{\prime}$ and $x, y \in X^{\prime}$. Hence, $(\tilde{T}, \tilde{\lambda})$ explains $\varepsilon^{\prime}$; and therefore, $\varepsilon^{\prime}$ is a symmetrized Fitch map.

Proposition 3.13. Let $\varepsilon:[X \times X]_{i r r} \rightarrow \mathcal{P}(M)$ be a symmetrized Fitch map. Then, for every color $m \in M$ the following equivalent statements are satisfied:

1. $\mathcal{G}_{m}(\varepsilon)$ does not contain a $K_{1}+K_{2}$ as an induced subgraph.

2. For every three pairwise distinct $a, b, c \in X$ with $m \notin \varepsilon(a, b)$ and $m \notin \varepsilon(b, c)$, we have $m \notin$ $\varepsilon(a, c)$.

3. $\mathcal{N}_{\neg m}[\varepsilon]$ is a partition of $X$.

4. $\mathcal{G}_{m}(\varepsilon)$ is a complete multi-partite graph, where the neighborhoods in $\mathcal{N}_{\neg m}[\varepsilon]$ form precisely the maximal independent sets in $\mathcal{G}_{m}(\varepsilon)$.

5. For every $N \in \mathcal{N}_{\neg m}[\varepsilon]$, we have $N=N_{\neg m}[y]$ if and only if $y \in N$.

Proof: Let $\varepsilon:[X \times X]_{\text {irr }} \rightarrow \mathcal{P}(M)$ be a symmetrized Fitch map, and let $m \in M$ be an arbitrary color. Then, Lemma 3.12 implies that the map $\varepsilon^{\prime}:[X \times X]_{\text {irr }} \rightarrow \mathcal{P}(\{m\})$ with $\varepsilon^{\prime}(x, y):=\varepsilon(x, y) \cap\{m\}$ for every $(x, y) \in[X \times X]_{\text {irr }}$ is a (monochromatic) symmetrized Fitch map. In particular, $\mathcal{N}_{\neg m}[\varepsilon]=\mathcal{N}_{\neg m}\left[\varepsilon^{\prime}\right]$. Hence, we can apply Lemma 3.5 and Prop.3.6 to conclude that the Statements (1), (2) and (3) are satisfied and equivalent.

We continue by showing the equivalence between Statement (3) and (4). To this end, observe first that Lemma $3.5(1,3)$ and Proposition $3.6(1,3)$ directly imply that $\mathcal{G}_{m}(\varepsilon)$ is a complete multi-partite graph if and only if $\mathcal{N}_{\neg m}[\varepsilon]$ is a partition of $X$. Note, each complete multi-partite graph is, by definition, determined by its maximal independent sets. It remains to show that the neighborhoods in $\mathcal{N}_{\neg m}[\varepsilon]$ are precisely the maximal independent sets of $\mathcal{G}_{m}(\varepsilon)$. Let $N_{\neg m}[y] \in \mathcal{N}_{\neg m}[\varepsilon]$. By definition, for all $a, b \in$ $N_{\neg m}[y]$ we have $m \notin \varepsilon(a, y)$ and $m \notin \varepsilon(b, y)$. Hence, Statement (2) implies that $m \notin \varepsilon(a, b)$. By definition of $\mathcal{G}_{m}(\varepsilon)$ neither of $\{a, y\},\{b, y\}$ and $\{a, b\}$ forms an edge in $\mathcal{G}_{m}(\varepsilon)$. Hence, $N_{\neg m}[y]$ is an independent set of $\mathcal{G}_{m}(\varepsilon)$. Assume, for contradiction, that $N_{\neg m}[y]$ is not a maximal independent set. Hence, there is a vertex $z \in V \backslash N_{\neg m}[y]$ such that $\{z, v\} \notin E\left(\mathcal{G}_{m}(\varepsilon)\right)$ for all $v \in N_{\neg m}[y]$. In particular, therefore, $\{z, y\} \notin E\left(\mathcal{G}_{m}(\varepsilon)\right)$ and thus, by definition of $\mathcal{G}_{m}(\varepsilon), m \notin \varepsilon(z, y)$. But then, $z \in N_{\neg m}[y]$; 


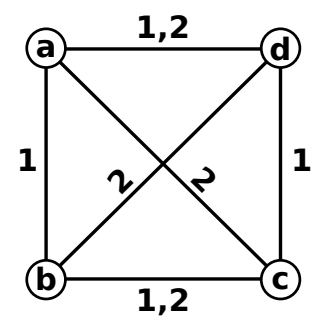

Fig. 5: Let $\varepsilon:[X \times X]_{\text {irr }} \rightarrow \mathcal{P}(M)$ be a symmetric map with $X:=\{a, b, c, d\}$ and $M:=\{1,2\}$, where for every distinct $x, y \in X$ and every $m \in M$ we have $m \in \varepsilon(x, y)$ if and only if the edge $\{x, y\}$ in the shown graph contains the label $m$. Then, $\varepsilon$ satisfies Prop. 3.6(1) and (2). However, $\varepsilon$ is not a Fitch map, see text for further details.

a contradiction. Therefore, the neighborhoods in $\mathcal{N}_{\neg m}[\varepsilon]$ are precisely the maximal independent sets of $\mathcal{G}_{m}(\varepsilon)$.

We continue with showing that Statement (3) and (5) are equivalent. First, suppose that Statement (3) is satisfied, and let $N \in \mathcal{N}_{\neg m}[\varepsilon]$. If $N=N_{\neg m}[y]$, then we have by definition $y \in N_{\neg m}[y]=N$. Conversely, if $y \in N$, then we have $y \in N \cap N_{\neg m}[y] \neq \emptyset$. Hence, since $\mathcal{N}_{\neg m}[\varepsilon]$ with $N, N_{\neg m}[y] \in$ $\mathcal{N}_{\neg m}[\varepsilon]$ is a partition of $X$, we conclude that $N=N_{\neg m}[y]$.

Next, we assume that Statement (5) is satisfied, and let $N, N^{\prime} \in \mathcal{N}_{\neg m}[\varepsilon]$ be two arbitrary neighborhoods. Since we have $y \in N_{\neg m}[y]$ for every $y \in X$, we conclude that every neighborhood is non-empty in $\mathcal{N}_{\neg m}[\varepsilon]$ and $\bigcup_{y \in X} N_{\neg m}[y]=X$. Moreover, let $N \cap N^{\prime} \neq \emptyset$. Hence, there is a vertex $y \in N \cap N^{\prime}$, and thus by Statement (5) we obtain $N=N_{\neg m}[y]=N^{\prime}$. The latter arguments together imply that $\mathcal{N}_{\neg m}[\varepsilon]$ is a partition of $X$, and thus Statement (3) is satisfied.

We will need to define certain sets of subsplits associated with the complementary neighborhoods of $\varepsilon$.

Definition 3.14. For a symmetric map $\varepsilon:[X \times X]_{\text {irr }} \rightarrow \mathcal{P}(M)$ we define the following sets:

- The m-subsplit system of $\varepsilon$ is $\mathcal{S}_{m}(\varepsilon):=\left\{N \mid N^{\prime}: N, N^{\prime} \in \mathcal{N}_{\neg m}[\varepsilon]\right.$ and $\left.N \cap N^{\prime}=\emptyset\right\}$;

- The subsplit system of $\varepsilon$ is $\mathcal{S}(\varepsilon):=\bigcup_{m \in M} \mathcal{S}_{m}(\varepsilon)$; and

- the non-trivial subsplit system of $\varepsilon$ is $\mathcal{S}^{\star}(\varepsilon):=\left\{N\left|N^{\prime}: N\right| N^{\prime} \in \mathcal{S}(\varepsilon)\right.$ and $\left.|N|,\left|N^{\prime}\right| \geq 2\right\}$.

Clearly, if a set $\mathcal{S}$ of subsplits is compatible, then every subset $\mathcal{S}^{\prime} \subseteq \mathcal{S}$ is also compatible. $\mathcal{S}(\varepsilon)$ is compatible if and only if $\mathcal{S}^{\star}(\varepsilon)$ is compatible because every subsplit $N \mid N^{\prime} \in \mathcal{S}(\varepsilon) \backslash \mathcal{S}^{\star}(\varepsilon)$ is trivial and $\mathcal{S}^{\star}(\varepsilon) \subseteq \mathcal{S}(\varepsilon)$. For later reference we summarize the latter observation in the following

Lemma 3.15. Let $\varepsilon:[X \times X]_{i r r} \rightarrow \mathcal{P}(M)$ be a map. Then, $\mathcal{S}(\varepsilon)$ is compatible if and only if $\mathcal{S}^{\star}(\varepsilon)$ is compatible.

Before we provide our final characterization we observe that compatibility of $\mathcal{S}(\varepsilon)$ is a necessary condition for Fitch maps.

Lemma 3.16. Let $\varepsilon:[X \times X]_{i r r} \rightarrow \mathcal{P}(M)$ be a symmetrized Fitch map, and let $\mathcal{S}(\varepsilon)$ be the subsplit system of $\varepsilon$. Then, every edge-labeled tree $(T, \lambda)$ that explains $\varepsilon$ displays all subsplits in $\mathcal{S}(\varepsilon)$. 
Proof: Let $\varepsilon:[X \times X]_{\text {irr }} \rightarrow \mathcal{P}(M)$ be a symmetrized Fitch map, and let $(T, \lambda)$ be an arbitrary edgelabeled tree that explains $\varepsilon$. We denote by $T_{\mid L}$ the vertex-minimal (not necessarily phylogenetic) subtree of $T$ with leaf set $L \subseteq \mathcal{L}(T)$.

Assume for contradiction that there is a subsplit $N \mid N^{\prime} \in \mathcal{S}(\varepsilon)$ that is not displayed by $T$. Clearly, if $|N|=1$ or $\left|N^{\prime}\right|=1$, then $T$ displays $N \mid N^{\prime}$. Thus, we can assume that $|N|>1$ and $\left|N^{\prime}\right|>1$. Moreover, if none of the paths $P_{T}(a, b)$ and $P_{T}(c, d)$ with $a, b \in N$ and $c, d \in N^{\prime}$ intersect, then the two trees $T_{\mid N}$ and $T_{\mid N^{\prime}}$ are vertex disjoint, and thus, there would be an edge $e \in E(T)$ such that $N \subseteq \mathcal{L}\left(T_{1}\right)$ and $N^{\prime} \subseteq \mathcal{L}\left(T_{2}\right)$. Therefore, there are four leaves $a, b \in N$ and $c, d \in N^{\prime}$ such that the paths $P_{T}(a, b)$ and $P_{T}(c, d)$ intersect. Hence, there is a vertex $v \in V\left(P_{T}(a, b)\right) \cap V\left(P_{T}(c, d)\right)$. Proposition 3.13 (5), together with $a, b \in N$ and $c, d \in N^{\prime}$, implies that $a \in N=N_{\neg m}[b]$ and $c \in N^{\prime}=N_{\neg m}[d]$. This, together with the fact that $(T, \lambda)$ explains $\varepsilon$, implies that there is no $m$-edge on either of the paths $P_{T}(a, b)$ and $P_{T}(c, d)$. Since $v$ lies on both paths $P_{T}(a, b)$ and $P_{T}(c, d)$, there is no $m$-edge on the (sub)paths $P_{T}(a, v)$ and $P_{T}(v, d)$. Therefore, the path $P_{T}(a, d) \subseteq P_{T}(a, v) \cup P_{T}(v, d)$ cannot contain an $m$-edge. Now, Proposition 3.13 (5) and $a \in N$ imply that $N=N_{\neg m}[a]$. However, since $N \mid N^{\prime}$ is a subsplit, we have $N \cap N^{\prime}=\emptyset$, and therefore $d \notin N=N_{\neg m}[a]$. This, together with the fact that $(T, \lambda)$ explains $\varepsilon$, implies that there is an $m$-edge on the path $P_{T}(a, d)$; a contradiction. In summary, every subsplit $N \mid N^{\prime} \in \mathcal{S}(\varepsilon)$ is displayed by $T$.

Lemma 3.16, together with Lemma 3.15, immediately implies

Corollary 3.17. If $\varepsilon:[X \times X]_{i r r} \rightarrow \mathcal{P}(M)$ is a symmetrized Fitch map, then the subsplit sets $\mathcal{S}(\varepsilon)$ and $\mathcal{S}^{\star}(\varepsilon)$ are compatible.

Definition 3.18. Let $\varepsilon:[X \times X]_{\text {irr }} \rightarrow \mathcal{P}(M)$ be a symmetric map such that $\mathcal{S}(\varepsilon)$ is compatible. Then, we denote with $\left(T_{\varepsilon}, \lambda_{\varepsilon}\right)$ an edge-labeled tree that satisfies the following two conditions:

1. $T_{\varepsilon}$ displays every subsplit in $\mathcal{S}(\varepsilon)$; and

2. for every edge $e \in E\left(T_{\varepsilon}\right)$ we have

$$
\lambda_{\varepsilon}(e):=\left\{m \in M: \begin{array}{ll}
\text { (a) } e \text { is a splitting edge w.r.t. some } N \mid N^{\prime} \in \mathcal{S}_{m}(\varepsilon) \text { and } \\
\text { (b) for every } N \in \mathcal{N}_{\neg m}[\varepsilon] \text { and for every } x, y \in N \text { we have } e \notin E\left(P_{T_{\varepsilon}}(x, y)\right)
\end{array}\right\} .
$$

Lemma 3.19. $\varepsilon:[X \times X]_{i r r} \rightarrow \mathcal{P}(M)$ is a symmetrized Fitch map if it satisfies the following two conditions:

1. for every $m \in M$ the set $\mathcal{N}_{\neg m}[\varepsilon]$ forms a partition of $X$; and

2. $\mathcal{S}^{\star}(\varepsilon)$ is compatible.

In particular, $\left(T_{\varepsilon}, \lambda_{\varepsilon}\right)$ explains $\varepsilon$.

Proof: Let $\varepsilon:[X \times X]_{\text {irr }} \rightarrow \mathcal{P}(M)$ be a map that satisfies Conditions (1) and (2). Since $\mathcal{S}^{\star}(\varepsilon)$ is compatible, Lemma 3.15 implies that $\mathcal{S}(\varepsilon)$ is compatible. Hence, there is a tree $T_{\varepsilon}$ that displays every subsplit in $\mathcal{S}(\varepsilon)$. For $T_{\varepsilon}$ let $\lambda_{\varepsilon}: E\left(T_{\varepsilon}\right) \rightarrow \mathcal{P}(M)$ be the edge-labeling as specified in Def. 3.18 (2). Hence, we obtain an edge labeled-tree $\left(T_{\varepsilon}, \lambda_{\varepsilon}\right)$ that satisfies Def. 3.18. To show that $\varepsilon$ is a symmetrized Fitch map, it suffices to show that $\left(T_{\varepsilon}, \lambda_{\varepsilon}\right)$ explains $\varepsilon$. Thus, we must verify that for every two distinct 
leaves $x, y \in X$ we have $m \in \varepsilon(x, y)$ if and only if there is an $m$-edge on the path $P_{T_{\varepsilon}}(x, y)$. To this end, let $m \in M$ be an arbitrary color, and let $x, y \in X$ be two distinct arbitrary leaves.

First, suppose that $m \in \varepsilon(x, y)$. Then, we have $y \notin N_{\neg m}[x]$. This and $y \in N_{\neg m}[y]$ implies that $N_{\neg m}[x] \neq N_{\neg m}[y]$. Thus, since $\mathcal{N}_{\neg m}[\varepsilon]$ is a partition of $X$, it must hold that $N_{\neg m}[x] \cap N_{\neg m}[y]=\emptyset$. Therefore, by definition of $\mathcal{S}(\varepsilon)$, we have $N_{\neg m}[x] \mid N_{\neg m}[y] \in \mathcal{S}_{m}(\varepsilon) \subseteq \mathcal{S}(\varepsilon)$. Since $T_{\varepsilon}$ displays every subsplit in $\mathcal{S}(\varepsilon)$, there is a splitting edge $e \in E\left(T_{\varepsilon}\right)$ w.r.t. $N_{\neg m}[x] \mid N_{\neg m}[y]$. Hence, we have $N_{\neg m}[x] \subseteq$ $\mathcal{L}\left(T_{e, x}\right)$ and $N_{\neg m}[y] \subseteq \mathcal{L}\left(T_{e, y}\right)$, where $T_{e, x}$ and $T_{e, y}$ are the two connected components of $T_{\varepsilon} \backslash e$. We may assume w.l.og. that this splitting edge $e=\{v, w\}$ w.r.t. $N_{\neg m}[x] \mid N_{\neg m}[y]$ is chosen such that $v$ lies on the (unique) path $P_{T_{\varepsilon}}(w, x)$ and that $\left|V\left(T_{e, x}\right)\right|$ is minimal among all such splitting edges w.r.t. $N_{\neg m}[x] \mid N_{\neg m}[y]$.

There are two cases, either $\left|V\left(T_{e, x}\right)\right|=1$ or $\left|V\left(T_{e, x}\right)\right|>1$. First, suppose that $\left|V\left(T_{e, x}\right)\right|=1$. This is if and only if $\mathcal{L}\left(T_{e, x}\right)=V\left(T_{e, x}\right)=\{v\}=\{x\}$. Therefore, $x \in N_{\neg m}[x] \subseteq \mathcal{L}\left(T_{e, x}\right)=\{x\}$ implies $\{x\}=N_{\neg m}[x]$, and thus, $\left|N_{\neg m}[x]\right|=1$. Assume for contradiction that there is an $N \in \mathcal{N}_{\neg m}[\varepsilon]$ with $x^{\prime}, y^{\prime} \in N$ such that $e \in E\left(P_{T_{\varepsilon}}\left(x^{\prime}, y^{\prime}\right)\right)$. Since the path $P_{T_{\varepsilon}}\left(x^{\prime}, y^{\prime}\right)$ contains an edge, we conclude that $x^{\prime} \neq y^{\prime}$, and thus, $|N| \geq 2$. Therefore, $N \neq N_{\neg m}[x]$. Now, $N, N_{\neg m}[x] \in \mathcal{N}_{\neg m}[\varepsilon]$, which forms a partition of $X$, implies that $N \cap N_{\neg m}[x]=\emptyset$. However, since $e=\{v, w\}=\{x, w\}$ is an outer edge, we conclude that $x \in\left\{x^{\prime}, y^{\prime}\right\} \subseteq N$. Thus, $x \in N \cap N_{\neg m}[x] \neq \emptyset$; a contradiction. Hence, Condition (2b) in Def. 3.18 is satisfied. Thus, by construction of $\lambda_{\varepsilon}$, we have $m \in \lambda_{\varepsilon}(e)$. Since $e$ is an edge of the path $P_{T_{\varepsilon}}(x, y)$ there is an $m$-edge in $P_{T_{\varepsilon}}(x, y)$.

Otherwise, if $\left|V\left(T_{e, x}\right)\right|>1$ and thus $\left|\mathcal{L}\left(T_{e, x}\right)\right|>1$, then the minimality of $\left|V\left(T_{e, x}\right)\right|$ implies that there are two leaves $x^{\prime}, x^{\prime \prime} \in N_{\neg m}[x]$ such that $v \in V\left(P_{T_{\varepsilon}}\left(x^{\prime}, x^{\prime \prime}\right)\right)$.

Now, assume for contradiction that $e$ is not an $m$-edge. Since $e$ satisfies Condition (2a) in Def. 3.18, it can therefore, not satisfy Condition (2b) in Def. 3.18. Hence, there is a neighborhood $N^{\prime} \in \mathcal{N}_{\neg m}[\varepsilon]$ with $z^{\prime}, z^{\prime \prime} \in N^{\prime}$ such that $e \in E\left(P_{T_{\varepsilon}}\left(z^{\prime}, z^{\prime \prime}\right)\right)$. This, together with $e=\{v, w\}$, implies $v \in V\left(P_{T_{\varepsilon}}\left(x^{\prime}, x^{\prime \prime}\right)\right) \cap$ $V\left(P_{T_{\varepsilon}}\left(z^{\prime}, z^{\prime \prime}\right)\right)$. Since one of the leaves in $\left\{z^{\prime}, z^{\prime \prime}\right\} \subseteq N^{\prime}$ is not contained in $T_{e, x}$ and since $N_{\neg m}[x] \subseteq$ $\mathcal{L}\left(T_{e, x}\right)$, we have $N^{\prime} \neq N_{\neg m}[x]$. Since $\mathcal{N}_{\neg m}[\varepsilon]$ is a partition of $X$, it must hold that $N^{\prime} \cap N_{\neg m}[x]=\emptyset$. Therefore, $N^{\prime} \mid N_{\neg m}[x] \in \mathcal{S}_{m}(\varepsilon) \subseteq \mathcal{S}(\varepsilon)$. However, $v \in V\left(P_{T_{\varepsilon}}\left(x^{\prime}, x^{\prime \prime}\right)\right) \cap V\left(P_{T_{\varepsilon}}\left(z^{\prime}, z^{\prime \prime}\right)\right)$, together with $x^{\prime}, x^{\prime \prime} \in N_{\neg m}[x]$ and $z^{\prime}, z^{\prime \prime} \in N^{\prime}$, implies that the subsplit $N^{\prime} \mid N_{\neg m}[x] \in \mathcal{S}(\varepsilon)$ is not displayed by $T_{\varepsilon}$; a contradiction. Therefore, $e$ is an $m$-edge that lies on the path $P_{T_{\varepsilon}}(x, y)$.

It remains to show that the existence of an $m$-edge on the path $P_{T_{\varepsilon}}(x, y)$ implies $m \in \varepsilon(x, y)$. Using contraposition, assume that $m \notin \varepsilon(x, y)$, and thus $x, y \in N_{\neg m}[x] \in \mathcal{N}_{\neg m}[\varepsilon]$. For every edge $e \in$ $E\left(P_{T_{\varepsilon}}(x, y)\right)$, Condition (2b) in Def. 3.18 is violated. Hence, for all $e \in E\left(P_{T_{\varepsilon}}(x, y)\right)$, we have by construction of $\lambda_{\varepsilon}$ that $m \notin \lambda_{\varepsilon}(e)$. Thus, $P_{T_{\varepsilon}}(x, y)$ does not contain an $m$-edge, which completes the proof.

In summary, we have shown that $\left(T_{\varepsilon}, \lambda_{\varepsilon}\right)$ explains $\varepsilon$. Therefore, $\varepsilon$ is a symmetrized Fitch map.

The characterization of Fitch maps, which is summarized in Theorem 3.20 , follows now directly from Proposition 3.13 (3), Corollary 3.17 and Lemma 3.19

Theorem 3.20. A symmetric map $\varepsilon:[X \times X]_{\text {irr }} \rightarrow \mathcal{P}(M)$ is a symmetrized Fitch map if and only if

1. for every $m \in M$ the set $\mathcal{N}_{\neg m}[\varepsilon]$ forms a partition of $X$; and

2. $\mathcal{S}^{\star}(\varepsilon)$ is compatible.

For later reference we state here a simple consequence of Theorem 3.20 
Corollary 3.21. A symmetric map $\varepsilon:[X \times X]_{\text {irr }} \rightarrow \mathcal{P}(M)$, where $\mathcal{N}_{\neg m}[\varepsilon]$ forms a partition of $X$ for every $m \in M$, is a symmetrized Fitch map if and only if $\mathcal{S}^{\star}(\varepsilon)$ is compatible.

By Proposition 3.13, for every symmetrized Fitch map, $\varepsilon:[X \times X]_{\text {irr }} \rightarrow \mathcal{P}(M)$ the graph $\mathcal{G}_{m}(\varepsilon)$ is a complete multi-partite graph for each $m \in M$, where the neighborhoods in $\mathcal{N}_{\neg m}[\varepsilon]$ form precisely the maximal independent sets in $\mathcal{G}_{m}(\varepsilon)$. By Corollary 3.11. each monochromatic symmetrized Fitch map (and thus, the undirected Fitch graph $\mathcal{G}_{m}(\varepsilon)$ ) can be explained by unrooted trees $\left(T_{m}, \lambda_{m}\right)$ of diameter $\operatorname{diam}\left(T_{m}\right) \leq 4$, see Figures 3 and 4 for an example. The compatibility problem for $\varepsilon$ is thus related to the supertree problem for a collection of trees $T_{m}$ with diameter at most 4 , one for each color $m$. The problems do not appear to be equivalent, however, since $\mathcal{N}_{\neg m}[\varepsilon]$ does not uniquely determine a (leastresolved) tree that explains the corresponding Fitch graph.

\section{Complexity Results}

Since monochromatic symmetrized Fitch maps are characterized in terms of complete multi-partite graphs they can be recognized in polynomial time, cf. Hellmuth et al. (2018b). "Non-symmetrized" (not necessarily monochromatic) Fitch maps can also be recognized in polynomial time, cf. Hellmuth et al. (2020); Hellmuth (2019). However, as we shall show below, the recognition of symmetrized Fitch maps is NPcomplete, in general. More precisely, we consider the following decision problem.

\section{Problem (Symm-Fitch Recognition).}

Input: $\quad$ A symmetric map $\varepsilon:[X \times X]_{\mathrm{irr}} \rightarrow \mathcal{P}(M)$.

Question: Is $\varepsilon$ a symmetrized Fitch map, i.e., is there an edge-labeled tree $(T, \lambda)$ that explains $\varepsilon$ ?

In order to prove NP-completeness, we use a reduction from the following NP-complete problem Steel (1992a).

\section{Problem (QuARTET COMPATIBILITY).}

Input: $\quad$ A set $Q$ of quartets on $X$.

Question: Is $Q$ compatible?

Proposition 4.1 (Steel, 1992a, Thm. 1)). Quartet Compatibility is NP-complete.

Theorem 4.2. Symm-Fitch Recognition is NP-complete.

Proof: Clearly, Symm-Fitch Recognition $\in$ NP, since we can test in polynomial time whether a given edge-labeled tree $(T, \lambda)$ indeed explains $\varepsilon$.

Let $\mathcal{Q}=\left\{q_{1}, q_{2}, \ldots, q_{|Q|}\right\}$ be an arbitrary set of quartets on $X$. Now, we construct a map $\varepsilon$ : $[X \times$ $X]_{\text {irr }} \rightarrow \mathcal{P}(M)$ with $M=\{1,2, \ldots,|\mathcal{Q}|\}$ such that for every $(x, y) \in[X \times X]_{\text {irr }}$ we have

$$
\varepsilon(x, y):=\left\{i \in M: q_{i}=a b \mid c d \text { and }\{x, y\} \notin\{\{a, b\},\{c, d\}\}\right\} .
$$

By construction of $\varepsilon$ we have for every $q_{i}=a b \mid c d \in \mathcal{Q}$ :

$$
\begin{aligned}
& N_{\neg i}[a]=N_{\neg i}[b]=\{a, b\}, \\
& N_{\neg i}[c]=N_{\neg i}[d]=\{c, d\}, \text { and } \\
& N_{\neg i}[y]=\{y\} \text { for every } y \in X \backslash\{a, b, c, d\} .
\end{aligned}
$$


Hence, $\mathcal{N}_{\neg i}[\varepsilon]$ is a partition of $X$ for every color $i \in M$. Now, we continue to show that $\mathcal{Q}=\mathcal{S}^{\star}(\varepsilon)$. If $q_{i}=a b \mid c d \in \mathcal{Q}$ then, by construction of $\varepsilon$, we have $a b\left|c d=N_{\neg i}[a]\right| N_{\neg i}[c] \in \mathcal{S}^{\star}(\varepsilon)$. Conversely, if $a b \mid c d \in \mathcal{S}^{\star}(\varepsilon)$, then there is a color $i \in M$ such that $N_{\neg i}[a]=\{a, b\}$ and $N_{\neg i}[c]=\{c, d\}$. This and the construction of $\varepsilon$ imply that $a b \mid c d=q_{i} \in \mathcal{Q}$. Thus, we have $\mathcal{Q}=\mathcal{S}^{\star}(\varepsilon)$. Since $\mathcal{N}_{\neg i}[\varepsilon]$ is a partition of $X$ for every color $i \in M$, we can apply Cor. 3.21 to conclude that $\varepsilon$ is a symmetrized Fitch map if and only if $\mathcal{S}^{\star}(\varepsilon)=\mathcal{Q}$ is compatible.

Since deciding whether $\mathcal{Q}$ is compatible is NP-complete, see Prop. 4.1, we can conclude that deciding whether $\varepsilon$ is a symmetrized Fitch map is NP-hard. This, together with SYMM-Fitch RECOGNITION $\in$ NP, implies that SYMM-Fitch RECOGNITION is NP-complete.

We note in passing that Theorem 4.2 implies that there is no characterization of Fitch maps in terms of a finite set of forbidden subgraphs (unless $P=N P$ ).

\section{Summary and Outlook}

In this contribution, we have characterized a class of symmetric maps $\varepsilon:[X \times X]_{\text {irr }} \rightarrow \mathcal{P}(M)$, or equivalently, sets of (not necessarily disjoint) symmetric binary relations $R_{1}, \ldots R_{|M|}$ that arise in a natural way from edge-labeled trees with a set of "colors". The symmetrized Fitch maps are those for which $\varepsilon(x, y)$ is the set of colors encountered along the unique path connecting $x$ and $y$ in $T$. In the monochromatic cases $|M|=1$ there is only a single relation $R_{1}$ (or graph). As already shown by Hellmuth et al. (2018b), $R_{1}$ is symmetrized Fitch relation if and only if it is a complete multi-partite graph. Here we provide an alternative characterization in terms of complementary neighborhoods. Restricted symmetrized Fitch maps assign at most one color to each pair $(x, y)$, i.e., $|\varepsilon(x, y)| \leq 1$. We found that these two classes coincide. Therefore, such maps can be recognized in polynomial time. In the general case, we obtained a series of necessary conditions as well as a characterization in terms of monochromatic "induced" submaps and certain subsplits defined by the complementary neighborhoods of $\varepsilon$ that must be displayed by every tree explaining $\varepsilon$, i.e., the subsplit system must be compatible. These result were utilized to show that the recognition of symmetrized Fitch maps is NP-complete.

Although we have obtained a comprehensive characterization interesting open questions remain. The complete multi-partite graphs are a subclass of the cographs, i.e., graphs that do not contain a path of length four as in induced subgraph Corneil et al. (1981, 1985). Cographs can be explained by vertexlabeled trees. In particular, the di-cograph structure Crespelle and Paul (2006) of non-symmetrized Fitch maps has been very helpful in the construction of efficient recognition algorithms Geiß et al. (2018) for the directed case. Since for every color $m \in M$ the graph-representation $\mathcal{G}_{m}(\varepsilon)$ of a symmetrized Fitch map $\varepsilon$ must be a complete multi-partite graph, $\mathcal{G}_{m}(\varepsilon)$ is a cograph. Clearly, this does not help directly for efficient recognition algorithms since the recognition problem is NP-complete. However, if we restrict our attention to maps $\varepsilon:[X \times X]_{\text {irr }} \rightarrow \mathcal{P}(M)$ that additionally satisfy the "triangle condition" $|\{\varepsilon(x, y), \varepsilon(x, z), \varepsilon(y, z)\}| \leq 2$ for every pairwise distinct $x, y, z \in X$, then we obtain the subclass of so-called unp 2-structures Hellmuth et al. (2017), which can be recognized in polynomial time. In future work we will investigate whether symmetrized Fitch map that satisfy this triangle condition can be recognized in polynomial time. Complementary, one may ask whether there are interesting constellations of complementary neighborhoods for which compatibility of $\mathcal{S}^{\star}(\varepsilon)$ can be checked efficiently, e.g. by the All Quartets Algorithm (Warnow, 2017, Sect. 5.2).

In Hellmuth et al. (2020), we characterized non-symmetrized " $k$-restricted" Fitch maps that can be explained by edge-labeled trees $(T, \lambda)$ with $|\lambda(e)| \leq k$ for every $e \in E(T)$ and some fixed integer 
$k$. This characterization was entirely based on the cardinality of complementary neighborhoods and the proof relied on the fact that the least-resolved tree for a non-symmetrized Fitch map is unique. However, finding a characterization for " $k$-restricted" symmetrized Fitch maps, seems to be quite difficult, since we cannot build upon the fact that least-resolved trees are unique for symmetrized Fitch maps (see Fig. 3 for a counterexample). Thus, it remains an open question if such restrictions may lead to deeper understanding of symmetrized Fitch maps and whether such maps can be recognized in polynomial time or not.

Real-life estimates of graphs are usually subject to measurement errors. Attempts to correct these estimates naturally leads to editing problem. In our setting, given a symmetric map $\varepsilon$, we are interested in a symmetrized Fitch map $\varepsilon^{\prime}$ that is "as close as possible" to $\varepsilon$. A natural distance measure is e.g. the sum of the symmetric differences of the edges of $\mathcal{G}_{m}(\varepsilon)$. In the light of Corollary 3.21 one may ask whether there is a connection between this "Fitch Map Editing" problem and the problem of finding a maximal subset of consistent quartets in $\mathcal{S}^{\star}(\varepsilon)$. Conversely, can one of the many heuristics for the MAXIMUM Quartet Consistency Problem (see Morgado and Marques-Silva (2010); Reaz et al. (2014) and the references therein) be adapted such that $\mathcal{N}_{\neg m}[\varepsilon]$ remains a partition for every $m \in M$ ?

\section{Acknowledgements}

We thank the anonymous referees for their constructive comments that helped to significantly improve the paper. In particular, their comments opened the avenue to characterize least- and minimally-resolved trees for monochromatic symmetrized Fitch relations.

\section{References}

S. Böcker and A. W. M. Dress. Recovering symbolically dated, rooted trees from symbolic ultrametrics. Adv. Math., 138:105-125, 1998. doi: 10.1006/aima.1998.1743.

D. Bryant and J. Lagergren. Compatibility of unrooted phylogenetic trees is FPT. Theor. Comp. Sci., 351: 296-302, 2006. doi: 10.1016/j.tcs.2005.10.033.

P. Buneman. The recovery of trees from measures of dissimilarity. In Mathematics the the Archeological and Historical Sciences, pages 387-395, United Kingdom, 1971. Edinburgh University Press.

T. Calamoneri and B. Sinaimeri. Pairwise compatibility graphs: A survey. SIAM Review, 58:445-460, 2016. doi: $10.1137 / 140978053$.

D. G. Corneil, H. Lerchs, and L. Steward Burlingham. Complement reducible graphs. Discr. Appl. Math., 3:163-174, 1981. doi: 10.1016/0166-218X(81)90013-5.

D. G. Corneil, Y. Perl, and L. K. Stewart. A linear recognition algorithm for cographs. SIAM J. Computing, 14:926-934, 1985. doi: 10.1137/0214065.

C. Crespelle and C. Paul. Fully dynamic recognition algorithm and certificate for directed cographs. Discr. Appl. Math., 154:1722-1741, 2006. doi: 10.1016/j.dam.2006.03.005.

A. Ehrenfeucht and G. Rozenberg. Theory of 2-structures, part II: Representation through labeled tree families. Theor. Comp. Sci., 70:305-342, 1990. doi: 10.1016/0304-3975(90)90130-A. 
D. Fernández-Baca. The perfect phylogeny problem. In X. Z. Cheng and D. Z. Du, editors, Steiner Trees in Industry, volume 11 of Combinatorial Optimization, pages 203-234. Springer, Boston, MA, 2001. doi: 10.1007/978-1-4613-0255-1_6.

W. M. Fitch. Toward defining the course of evolution: minimum change for a specified tree topology. Systematic Zoology, 20:406-416, 1971. doi: 10.1093/sysbio/20.4.406.

W. M. Fitch. Homology: a personal view on some of the problems. Trends Genet., 16:227-231, 2000. doi: $10.1016 / \mathrm{S} 0168-9525(00) 02005-9$.

M. Geiß, J. Anders, P. F. Stadler, N. Wieseke, and M. Hellmuth. Reconstructing gene trees from Fitch's xenology relation. J. Math. Biol., 77:1459-1491, 2018. doi: 10.1007/s00285-018-1260-8.

M. Hellmuth. Generalized Fitch graphs: Edge-labeled graphs that are explained by edge-labeled trees. Discr. Appl. Math., 267:1-11, 2019. doi: 10.1016/j.dam.2019.06.015.

M. Hellmuth and C. R. Seemann. Alternative characterizations of Fitch's xenology relation. J. Math. Biol., 79:969-986, 2019. doi: 10.1007/s00285-019-01384-х.

M. Hellmuth, P. F. Stadler, and N. Wieseke. The mathematics of xenology: Di-cographs, symbolic ultrametrics, 2-structures and tree-representable systems of binary relations. J. Math. Biol., 75:299-237, 2017. doi: 10.1007/s00285-016-1084-3.

M. Hellmuth, M. Hernandez-Rosales, Y. Long, and P. F. Stadler. Inferring phylogenetic trees from the knowledge of rare evolutionary events. J. Math. Biol., 76:1623-1653, 2018a. doi: 10.1007/ s00285-017-1194-6.

M. Hellmuth, Y. Long, M. Geiß, and P. F. Stadler. A short note on undirected Fitch graphs. Art Discr. Appl. Math., 1:P1.08, 2018b. doi: 10.26493/2590-9770.1245.98c.

M. Hellmuth, C. R. Seemann, and P. F. Stadler. Generalized Fitch graphs II: Sets of binary relations that are explained by edge-labeled trees. Discr. Appl. Math., 283:495-511, 2020. doi: 10.1016/j.dam.2020. 01.036 .

S. Kannan and T. Warnow. A fast algorithm for the computation and enumeration of perfect phylogenies. SIAM J. Comput., 26:1749-1763, 1997. doi: 10.1137/S0097539794279067.

A. Morgado and J. Marques-Silva. Combinatorial optimization solutions for the Maximum Quartet Consistency Problem. Fundamenta Informaticae, 102:363-389, 2010. doi: 10.3233/FI-2010-311.

R. Reaz, M. S. Bayzid, and M. S. Rahman. Accurate phylogenetic tree reconstruction from quartets: a heuristic approach. PLoS One, 9:e104008, 2014. doi: 10.1371/journal.pone.0104008.

D. Schaller, M. Lafond, P. F. Stadler, N. Wieseke, and M. Hellmuth. Indirect identification of horizontal gene transfer. Technical Report arXiv:2012.08897, arXiv, 2020.

C. Semple and M. Steel. Phylogenetics. Oxford University Press, New York, 2003. ISBN 978-0-19850942-4. 
M. Steel. The complexity of reconstructing trees from qualitative characters and subtrees. J. Classification, 9:91-116, 1992a. doi: 10.1007/BF02618470.

M. A. Steel. The complexity of reconstructing trees from qualitative characters and subtrees. J. Classification, 9:91-116, 1992b. doi: 10.1007/BF02618470.

T. Warnow. Computational Phylogenetics: An Introduction to Designing Methods for Phylogeny Estimation. Cambridge University Press, Cambridge, UK, 2017.

I. E. Zverovich. Near-complete multipartite graphs and forbidden induced subgraphs. Discrete Math., 207:257-262, 1999. doi: 10.1016/S0012-365X(99)00050-3. 